\title{
A case-control study of cataract in Oxfordshire: some risk factors
}

\author{
RUTH VAN HEYNINGEN AND JOHN J HARDING \\ From the Nuffield Laboratory of Ophthalmology, University of Oxford, Walton Street, Oxford OX2 6AW
}

SUMmaRY Three hundred patients with cataract and 609 control subjects with the same age-sex distribution were interviewed in a study of cataract in Oxfordshire, England. The risks associated with severe diarrhoea, glaucoma, and work on a military base have been demonstrated. Population attributable risks were calculated for those and other risk factors.

Cataract is the major cause of blindness worldwide and yet it is frequently stated that most cataracts are of unknown aetiology. In the Western world it usually occurs late in life and has therefore been called senile cataract. In some third world countries it is more prevalent than in the West and develops at an earlier age. Our suggestion that diarrhoea might be an important factor in these countries ${ }^{1}$ has been supported by the results of a careful study from India. ${ }^{2}$ Several risk factors for cataract, including diabetes, glaucoma, and high levels of plasma glucose and urea, were identified in a study in Edinburgh, ${ }^{3}$ but they did not include diarrhoea. We have conducted a similar study in Oxfordshire with particular interest in diarrhoea, medical and drug history, smoking, drinking, and place of work. It was necessary to obtain information on past events and practices, because cataract may take many years to develop following exposure to a risk factor. Some results of this survey have been published in two short papers. ${ }^{45}$

Our aim was to identify major risk factors for cataract of any type. Our definition of cataract was such cases as required surgery.

\section{Subjects and methods}

Cases were those patients aged between 50 and 79 years admitted to the Oxford Eye Hospital for cataract extraction. Three hundred cases were interviewed on the ward. None refused.

The Oxford Eye Hospital is a National Health

Correspondence to Dr J J Harding.
Service (NHS) hospital serving the people of Oxfordshire and parts of Berkshire and Buckinghamshire. It is the only NHS eye hospital in this area. The hospital notes were not looked at before the interview, so it would not be possible to select particular groups (for example, those with arthritis) for interview. There were no other selection criteria apart from age (50-79).

\section{CONTROLS}

Four groups of controls were recruited. The first group consisted of inpatients at the Ear, Nose, and Throat Department and patients in the Dermatology Department (Slade Hospital). They were interviewed in hospital after being asked if they had cataract. Any giving a positive response were excluded. Subjects aged 50-79 years were recruited, but towards the end of the study recruitment concentrated on older subjects so that the age-sex distribution matched the cases. These departments are also NHS hospitals coming under the same area authority and serving essentially the same population (563000) as the Oxford Eye Hospital. As about $95 \%$ of the British population is served by the NHS, patients in these hospitals are representative of the population at large within the same region. The ENT department is one minute's walk from the Oxford Eye Hospital. Most of these hospital patients had been admitted for minor surgery and in that sense also were comparable to cases. The dermatology department is also in Oxford.

The other three control groups were identified from the age-sex registers of local general practitioners. This made it possible to match the age-sex distribution to that of cases (Table 1). These com- 
Table 1 Age-sex distribution of the cases and the different control groups

\begin{tabular}{|c|c|c|c|c|c|c|}
\hline Group & \multicolumn{6}{|c|}{ Age group } \\
\hline \multicolumn{7}{|c|}{$\begin{array}{l}\text { (a) Percentage of females of each group in each age group (total } \\
\text { number }=507 \text { ) }\end{array}$} \\
\hline & $50-54$ & $55-59$ & $60-64$ & $65-69$ & $70-74$ & $75-79$ \\
\hline 1 & $6 \cdot 2$ & 8.6 & $18 \cdot 5$ & $19 \cdot 1$ & $24 \cdot 1$ & $23 \cdot 5$ \\
\hline 2 & $6 \cdot 1$ & $10 \cdot 6$ & $18 \cdot 2$ & $22 \cdot 7$ & $22 \cdot 7$ & $19 \cdot 7$ \\
\hline 5 & $6 \cdot 1$ & $8 \cdot 1$ & $21 \cdot 2$ & $17 \cdot 2$ & $23 \cdot 2$ & $24 \cdot 2$ \\
\hline 6 & $6 \cdot 7$ & $7 \cdot 9$ & $20 \cdot 2$ & $16 \cdot 9$ & $23 \cdot 6$ & $24 \cdot 7$ \\
\hline 7 & $6 \cdot 6$ & $7 \cdot 7$ & $17 \cdot 6$ & $17 \cdot 6$ & $28 \cdot 6$ & $22 \cdot 0$ \\
\hline
\end{tabular}

(b) Percentage of males of each group in each age group (total number $=402$ )

\begin{tabular}{lllllll} 
& $50-54$ & $55-59$ & $60-64$ & $65-69$ & $70-74$ & $75-79$ \\
1 & $5 \cdot 8$ & $13 \cdot 8$ & $18 \cdot 1$ & $18 \cdot 8$ & $23 \cdot 2$ & $20 \cdot 3$ \\
2 & $5 \cdot 9$ & $15 \cdot 7$ & $17 \cdot 7$ & $17 \cdot 7$ & $23 \cdot 5$ & $19 \cdot 6$ \\
5 & $6 \cdot 1$ & $10 \cdot 6$ & $16 \cdot 7$ & $18 \cdot 2$ & $25 \cdot 8$ & $22 \cdot 7$ \\
6 & $5 \cdot 3$ & $12 \cdot 0$ & $17 \cdot 3$ & $22 \cdot 7$ & $22 \cdot 7$ & $20 \cdot 0$ \\
7 & $4 \cdot 2$ & $16 \cdot 7$ & $19 \cdot 4$ & $15 \cdot 3$ & $23 \cdot 6$ & $20 \cdot 8$ \\
\hline
\end{tabular}

Group 1 is the cataract group. Groups 2, 5, 6, 7 are the four control groups.

There were $300,117,165,164$, and 163 subjects respectively in each group. The mean ages were between $67 \cdot 1$ and $67 \cdot 8$ years for each of the five groups.

munity controls were interviewed at home a few days after a warning letter. The three general practice areas were chosen to give a good socioeconomic spread. Two were urban and one covered a nearby large village. Interviews of cases and controls overlapped. Under the NHS $95 \%$ of the British population are registered with general practitioners, who form the first source of health care, and hence their age-sex registers are a useful way of identifying controls.

Our control groups included some people with minor opacities of the lens, as we excluded only those who had been told that they had cataract. Such opacities were relatively trivial compared with the cataracts in the cases. (In the chosen age range most people have such opacities ${ }^{6}$ and to exclude them would be to restrict the controls to a small subgroup of the population.)

A total of 609 controls were interviewed. Of potential controls about $4 \%$ had cataract, while $5 \%$ refused or were too ill to interview or spoke no English.

\section{QUESTIONNAIRE}

The cases and controls were interviewed in exactly the same way except that potential controls were asked if they had cataract. Informed consent was obtained. Each subject was given a code number, the first digit identifying the control group or case. No other identifier was entered into the computer. Age, sex, weight, and height were recorded. All subjects were asked where they had worked and what occupa- tions they had followed. There were questions on general medical history and ocular history. All were asked if they were short-sighted as a child and further prompted by being asked if they wore spectacles as a child and then what the spectacles were for. Only when the interviewer $(\mathrm{RvH})$ was sure were they counted as myopic when young. ${ }^{4}$ They were asked if they had ever had severe diarrhoea lasting four days or more. If they had, the time since the last attack, and how attacks were treated, was recorded. They were asked what drugs they had taken regularly for more than four months at any time in the past. Finally, they were asked their current and past smoking and drinking habits.

DATA ANALYSIS

Data were entered into the University's VAX computer and analysed by the $\chi^{2}$ test for categorical variables and Student's $t$ test for continuous variables, mostly by means of the SAS statistical package. The data entered into the computer consisted of 66 digits, less than a single line, for each of the 909 subjects. Odds ratios (cross product ratios) were computed from contingency tables and are reported as valid estimates of relative risk. Thus a reported relative risk of 2 for a particular risk factor implies that the exposed group are twice as likely to end up having cataract extraction than those who have had no exposure (or least exposure) to that risk factor.

Confidence intervals were calculated as described in the SAS User's Guide: Statistics version 5. Each factor investigated was first tested on all 300 cases and 609 controls, but interesting results were subsequently studied in subclasses by sex, age group, etc.

\section{Results}

After the data file had been checked for obvious errors the comparability of the control groups was examined. There were no significant differences between the four control groups in the proportion of subjects reporting no serious illness; hypertension; diabetes; serious illness other than diabetes, renal failure, and hypertension; aspirin consumption; or unknown drugs. Therefore all controls were pooled for most analyses.

At the start of the study we knew there was a possibility of recall bias, especially between hospital and community groups, but the comparisons between the different control groups indicate that little if any recall bias has occurred. Vague categories such as 'other illness' and 'other drug' showed no difference between cases and controls.

Some results of this case-control survey have been published, including the exploration of two risk 
Table 2 Severe diarrhoea as a risk factor for cataract in septuagenarians: contingency table

\begin{tabular}{lccc}
\hline & Controls & Cases & Total \\
\hline Severe diarrhoea & 14 & 14 & 28 \\
Without severe diarrhoea & 268 & 123 & 391 \\
Total & 282 & 137 & 419 \\
Percentage positive & 5.0 & $10 \cdot 2$ & \\
\hline
\end{tabular}

$\chi^{2}$ test $p=0 \cdot 043$; relative risk $=2 \cdot 18 ; 95 \%$ confidence limits $1 \cdot 01$ to $4 \cdot 7$.

factors, diabetes and myopia, showing females to be particularly susceptible ${ }^{4}$ and the identification of aspirin-like analgesics as protective factors against cataract. ${ }^{5}$

\section{SEVERE DIARRHOEA}

Severe diarrhoea appeared as a marginal risk factor for cataract when comparing all cases and all controls $\left(\mathrm{p}=0.05 \chi^{2}\right.$ test; relative risk 1.6) but was found to be a stronger risk factor in those aged 70 to 79 years (Table 2). The increased risk in this older group probably relates to severe diarrhoea during military service in the Far East between 1939 and 1945. Nevertheless, severe diarrhoea is only a marginal risk factor in the Oxford population.

\section{RENAL FAILURE}

Renal failure appeared as a powerful risk factor with a relative risk of 12.4 (2.4 to 65$)$. With only one control out of 609 reporting renal failure compared with six out of 300 cases, the $\chi^{2}$ test was not applicable, but Fisher's exact test (2-tail) gave a probability of $0 \cdot 006$. Hence, renal failure was the most powerful risk we encountered in the total population, though affecting a small proportion of cases $(2 \%)$. Four of the seven renal failure patients were also insulin-taking diabetics, all of them cataract cases. None of them reported taking steroids. One case and one control reported severe diarrhoea.

GLA U COMA

Glaucoma appeared as a powerful risk factor

Table 3 Glaucoma as a risk factor for cataract

\begin{tabular}{lccc}
\hline & Controls & Cases & Total \\
\hline With glaucoma & 8 & 22 & 30 \\
Without glaucoma & 601 & 278 & 879 \\
Total & 609 & 300 & 909 \\
Percentage positive & $1 \cdot 3$ & $7 \cdot 3$ & \\
\hline
\end{tabular}

$\chi^{2}$ test $p<0.001 ;$ relative risk $=5.95 ; 95 \%$ confidence limits 2.6 to 13.5. $(\mathrm{p} \ll 0.001$ and relative risk 5.9) as shown in Table 3. The relative risks were high for each sex taken separately ( $5 \cdot 4$ for men, $6 \cdot 7$ for women), and high for both younger (3.6) and older (8.5) subjects. The cholinesterase inhibitors which were widely used to treat glaucoma are known to cause cataract ${ }^{7}$ but lenses of glaucoma patients opacify even without this therapy, ${ }^{8}$ and it appears that the cataract risk associated with glaucoma is caused by a combination of the disease itself and the treatment applied. ${ }^{69}$

It could be suggested that as the cataract cases are seen regularly in the Eye Hospital that their glaucoma is more likely to be known than that of the control patients. In theory this would tend to make any ocular condition appear as a risk factor. However, analysis of the data for all ocular problems apart from cataract, glaucoma, and myopia did not show this pooled category to be a risk factor. We consider therefore that the effect of a difference in ophthalmic care was negligible. Alternatively it could be suggested that cataract patients with glaucoma are more likely to have cataract extractions than those without glaucoma but similar arguments apply. It is most unlikely that the 6-fold increase in risk associated with glaucoma could be explained in this way.

\section{PLACE OF WORK}

All subjects were asked where they had worked, and a note was made of the three where they had worked for longest -in order. Certain major employers in the Oxford area were scored individually, for example, the University, the car industry, military bases, and publishing. Only one of these appeared as a significant risk factor and then only when it was the main place of work. Those whose major work place had been on a military base were more than twice as likely to have cataract (Table 4). Only one of these was female. For males only, the relative risk was 2.37 $(1.07$ to 5.3$)$ with $\mathrm{p}=0.03$.

The explanation for the association between cataract and work on a military base is not obvious. There is the possibility of encountering microwave (radar) and other equipment, but there is also the possibility that this group tended to drink and smoke more than others.

Table 4 Work on military bases and cataract

\begin{tabular}{lccc}
\hline & Controls & Cases & Total \\
\hline Military base & 13 & 14 & 27 \\
Elsewhere & 596 & 286 & 882 \\
Total & 609 & 300 & 909 \\
Percentage positive & $2 \cdot 1$ & $4 \cdot 7$ & \\
\hline
\end{tabular}

$\chi^{2}$ test $\mathrm{p}=0 \cdot 034 ;$ relative risk $=2 \cdot 24 ; 95 \%$ confidence limits 1.04 to $4 \cdot 84$. 
'Heavy beer drinking' and 'heavy smoking' were found to be risk factors for cataract in this study ${ }^{10}$ and could be responsible for the apparent danger of military bases. With only 14 military cases and 13 military controls (Table 4) stratification of beer drinkers and of cigarette smokers into four dose groups gave numbers too small for reliable analysis. Omitting only the 'heavy beer drinkers' (more than 2 pints $(950 \mathrm{ml})$ per day) from the analysis leaves work on a military base as a risk factor $(\mathrm{p}=0.021$, relative risk $=2 \cdot 5$ ), so beer drinking cannot explain this risk. However, after omission of 'heavy smokers' (defined in Harding and van Heyningen ${ }^{10}$ ) from the analysis the risk associated with military bases is no longer significant $(p=0 \cdot 089)$, though workers on military bases were still overrepresented among cases (apparent relative risk 1.97). Analysis for interactions by means of GLIM (Royal Statistical Society, Edinburgh) indicated that the risk associated with military bases was not the result of either heavy smoking or drinking.

\section{COMBINATION OF RISK FACTORS}

It is difficult to study interacting factors in any detail because the numbers reporting any two specific conditions are small. The two most powerful risk factors were glaucoma (Table 3 ) and diabetes. ${ }^{4}$ The four subjects in the study with both glaucoma and diabetes were all cases. Myopia early in life was also a risk factor. ${ }^{4}$ Myopia early in life was investigated because a myopic shift occurs at an early stage in the development of nuclear cataract. The four subjects reporting both myopia and diabetes were all cases. Of three subjects reporting myopia and glaucoma two were cases. Four of the five subjects reporting both severe diarrhoea and diabetes were cases. Three of the four subjects reporting both severe diarrhoea and glaucoma were cases. Although the data are limited, there is an impression that pairs of factors may be additive.

\section{ATTRIBUTABLE RISK}

It is of interest to note not only how powerful a risk factor may be but also, on the assumption of a causal relationship, how much cataract is attributable to each factor. We therefore calculated the population attributable risk percentage (PARP) for cataract by the method of Cole and MacMahon. ${ }^{11}$ The estimation of attributable risks from case-control studies depends on the incidence of the disease being low. Although the incidence of lens opacities is high in those aged 50 to 79,6 the incidence of cataract as we define it is low, less than 1 in 1000 total population of this region, therefore the calculation is valid. Of the risk factors identified in this study, diabetes had the highest attributable risk followed by early myopia
Table 5 Population attributable risk percentages (PARPS) for cataract

\begin{tabular}{ll}
\hline Factor & PARP (\%) \\
\hline Diabetes & $11 \cdot 9$ \\
Myopia & $6 \cdot 9$ \\
Glaucoma & $6 \cdot 1$ \\
Steroids & $4 \cdot 7$ \\
Severe diarrhoea & $4 \cdot 3$ \\
'Heavy smoking' & $2 \cdot 6$ \\
'Heavy drinking' & $2 \cdot 6$ \\
Work on military bases & $2 \cdot 5$ \\
Spironolactone & $2 \cdot 3$ \\
Nifedipine & $2 \cdot 0$ \\
Renal failure & $1 \cdot 8$ \\
\hline
\end{tabular}

Data for the drugs, smoking, and drinking from Harding and van Heyningen. ${ }^{10}$

PARPS are a measure of the proportions of cataract in Oxfordshire that is associated with each risk factor. They indicate the proportions of cataract that could be prevented by elimination of each risk factor.

and glaucoma (Table 5). The other risk factors accounted for less than $5 \%$ of cataract each.

\section{Discussion}

This survey was concerned with the risk factors for all cataracts. We did not divide cataracts into classes. Subtle classification rapidly diminishes the number available for analysis. Known risk factors such as diabetes do not produce a single morphology of cataract in the age range studied, and there is evidence for common pathways in mechanisms of cataract formation. ${ }^{6}$ The inclusion of some controls with minor lens opacities will have made it a little more difficult for us to identify risk factors but would not cause us to make type I errors (false positive results). Severe diarrhoea has been proposed as a major cause of cataract in tropical countries to account for their excess of cataract.' Subsequently the role of 'life-threatening diarrhoea' in cataract in Raipur, India, was established by a case-control study. ${ }^{2}$ It is perhaps surprising to find severe diarrhoea as a risk factor in the Oxford population, but it is not a powerful factor here. The relative risk was much greater in the study in India, where diarrhoea is both more common and more severe. ${ }^{2}$

The results of this study strengthen the view that cataract can be a complication of renal failure. ${ }^{6}$ This is the first study to identify the relative risk of cataract associated with renal failure, and it is notably high. Several factors that could be important in the aetiology of cataract in renal failure patients could also have a role in cataract following severe diarrhoea ${ }^{16}$ These include cyanate formed from urea and the osmotic stress. The suggestion that steroid 
therapy could have a role in cataract in renal failure can be ruled out in this study, because none of the renal failure patients reported taking steroids. Cyanate binds to lens proteins, producing several changes corresponding to those found in human cataract. These include conformational changes to the proteins and cross-linking. ${ }^{12}$ Cyanate causes opacification of lenses in vitro ${ }^{13}$ and in vivo. ${ }^{14}$

Glaucoma appeared as a powerful risk factor in this study, though none of the subjects reported taking cholinesterase inhibitors. The increased susceptibility to cataract is probably due to glaucoma itself or the surgery to alleviate it. The explanation for the association between cataract and work on a military base is not obvious. There is the possibility of encountering microwave (radar) and other equipment, but also this group tended to smoke more than others. It seemed possible that the result might have arisen because military personnel usually do not register with a general practitioner, but in the age range studied we had only ex-military people, and the relationship persisted in septuagenarians who had retired long before.

The data on pairs of risk factors are limited by the size of this study, but they support the idea that apparently distinct factors can act together in the formation of cataract. The only previous attempt to calculate attributable risks for cataract was in the study in India, where almost $40 \%$ of the cataract could be attributed to 'life-threatening diarrhoea'. ${ }^{2}$ In Oxfordshire no single risk factor accounts for so high a proportion (Table 5). In India, at least in Raipur, improved sanitation might in the long term eliminate up to $40 \%$ of the cataract burden; it would have little effect in Oxford. Here a programme for decreasing cataract would have to look at a number of factors, none of them easily removed. Diabetes cannot be avoided, though one would hope that improved control of blood glucose would lessen the risk. Weale ${ }^{15}$ suggested that overcorrection of myopes in their late 20 s to maintain their lenses in a slightly accommodated state would decrease their excess risk of cataract.

The link between glaucoma and cataract may relate to the severity of the glaucoma or to the trauma of operations to alleviate glaucoma, so it will be difficult to eliminate this risk. Use of steroids will decline only as alternative drugs emerge. One would think that the risk of diarrhoea-related cataract would be diminishing rapidly in England, but our sewage system is falling into disrepair and the incidence of diarrhoeal disease is increasing rapidly in some areas.

We are grateful to Professor $\mathrm{N}$ Wald for advice and for help in planning the questionnaire. We thank Mr A J Bron, Drs R A E Spilling, J Ross, D Minassian, and G Edwards for helpful discussions. We are grateful to the consultants of the Oxford Eye Hospital, Ear, Nose and Throat Department and the Slade Hospital, and to local general practitioners for access to their patients. We thank the TFC Frost Charitable Trust for a grant for expenses.

\section{References}

1 Harding JJ, Rixon KC. Carbamylation of lens proteins; a possible factor in cataractogenesis in some tropical countries. Exp Eye Res 1980; 31: 567-71.

2 Minassian D, Mehra V, Jones BR. Dehydrational crises from severe diarrhoea or heatstroke and risk of cataract. Lancet 1984: i: $751-3$.

3 Clayton RM, Cuthbert J, Duffy J. Some risk factors associated with cataract in SE Scotland: a pilot study. Trans Ophthalmol Soc UK 1982; 102: 331-6.

4 van Heyningen R, Harding JJ. Risk factors for cataract: diabetes, myopia and sex. In: Courtois Y, Forette B, Knook D, eds. Modern trends in ageing research. Paris: INSERM/Libbey: in press.

5 van Heyningen $R$, Harding JJ. Do aspirin-like analgesics protect against cataract? A case-control study. Lancet 1986; i: 1111-3.

6 Harding JJ, Crabbe MJC. The lens: development, proteins, metabolism and cataract. In: Davson $\mathrm{H}$, ed. The eye. 3rd ed. London: Academic Press, 1984: 1B: 207-492.

7 Axelsson U, Holmberg A. The frequency of cataract after miotic therapy. Acta Ophthalmol (KGh) 1966; 44: 421-9.

8 Jones BR. Cataracta glaucomatosa and its role in the diagnosis of the acute glaucomas. Trans Ophthalmol Soc UK 1959; 79: 753-60.

9 Bartholomew RS, Clayton RM, Cuthbert J. Analysis of individual cataract patients and their lenses: preliminary observations on a population basis. In: Regnault $F$, Hockwin $O$, Courtois Y, eds. Ageing of the lens. Amsterdam: Elsevier-North Holland, 1980: 241-61.

10 Harding JJ, van Heyningen R. Drugs, including alcohol, that act as risk factors for cataract, and possible protection against cataract by aspirin-like analgesics and cyclopenthiazide. $\mathrm{Br} J$ Ophthalmol 1988; 72: 809-14.

11 Cole $\mathbf{P}$, MacMahon B. Attributable risk percent in case-control studies. Br J Prev Soc Med 1971; 25: 242-4.

12 Beswick HT, Harding JJ. Conformational changes induced in bovine lens $\alpha$-crystallin by carbamylation. Relevance to cataract. Biochem J 1984; 223: 221-7.

13 Crompton M, Rixon K, Harding JJ. Aspirin prevents carbamylation of soluble lens proteins and prevents cyanate-induced phase separation opacities in vitro: a possible mechanism by which aspirin could prevent cataract. Exp Eye Res 1985; 40: 297-311.

14 Nicholson DH, Harkness DR, Benson WE, Peterson CM Cyanate-induced cataracts in patients with sickle-cell hemoglobinopathies. Arch Ophthalmol 1976; 94: 927-30.

15 Weale $\mathbf{R}$. A note on a possible relation between refraction and a disposition for senile nuclear cataract. Br J Ophthalmol 1980; 64: 311-4.

Accepted for publication 30 July 1987. 